\title{
Molecular evolution of plasminogen activator inhibitor-1 functional stability
}

\author{
Mitchell B.Berkenpas' ${ }^{1}$, Daniel A.Lawrence ${ }^{2}$ \\ and David Ginsburg 1,2,3,4 \\ ${ }^{1}$ Howard Hughes Medical Institute, ${ }^{2}$ Department of Internal Medicine \\ and ${ }^{3}$ Department of Human Genetics, 4520 MSRB I, University of \\ Michigan, Ann Arbor, MI 48109-0650, USA \\ ${ }^{4}$ Corresponding author
}

Plasminogen activator inhibitor-1 (PAI-1) is a member of the serine protease inhibitor (serpin) supergene family and a central regulatory protein in the blood coagulation system. PAI-1 is unique among serpins in exhibiting distinct active and inactive (latent) conformations in vivo. Though the structure of latent PAI-1 was recently solved, the structure of the shortlived, active form of PAI-1 is not known. In order to probe the structural basis for this unique conformational change, a randomly mutated recombinant PAI-1 expression library was constructed in bacteriophage and screened for increased functional stability. Fourteen unique clones were selected, and shown to exhibit functional half-lives $\left(T_{1 / 2} \mathrm{~s}\right)$ exceeding that of wild-type PAI-1 by up to 72 -fold. The most stable variant $\left(T_{1 / 2}=145 \mathrm{~h}\right)$ contained four mutations. Detailed analysis of these four mutations, individually and in combination, demonstrated that the markedly enhanced functional stability of the parent compound mutant required contributions from all four substitutions, with no individual $T_{1 / 2}$ exceeding $6.6 \mathrm{~h}$. The functional stability of at least eight of the remaining 13 compound mutants also required interactions between two or more amino acid substitutions, with no single variant increasing the $T_{1 / 2}$ by $>10$-fold. The nature of the identified mutations implies that the unique instability of the PAI-1 active conformation evolved through global changes in protein packing and suggest a selective advantage for transient inhibitor function. Key words: fibrinolysis/protein stability/random mutagenesis/serine protease inhibitor/serpin

\section{Introduction}

Many complex intracellular and extracellular processes are regulated by interactions between proteases and their specific inhibitors (Vassalli et al., 1991; Vassalli and Pepper, 1994). Plasminogen activator inhibitor-1 (PAI-1), a member of the serpin gene family, regulates tissue-type and urokinase-type plasminogen activators (tPA and uPA). Deficiency of PAI-1 in humans results in a moderate bleeding disorder (Fay et al., 1992) and recent observations in mice genetically engineered to be deficient in both uPA and tPA suggest that plasminogen activation may have other important functions, in addition to its clearly defined role in hemostasis (Carmeliet et al., 1994).
All members of the serpin gene family are thought to share a common molecular mechanism, which has been the subject of intense study (Carrell et al., 1991; Lawrence et al., 1994b; Potempa et al., 1994; Wei et al., 1994; Stein and Carrell, 1995). The mobile serpin reactive center loop (RCL) serves as 'bait' for the target protease, leading to formation of a locked, inactive complex. The exceptionally high mobility of the PAI-1 RCL may account for the spontaneous decay of native PAI- 1 into a more structurally stable, inactive or 'latent' conformation, with a $T_{1 / 2}$ of $\sim 2 \mathrm{~h}$, under physiological conditions. The biologic significance of the PAI-1 latent conformation is currently unknown. Though other serpins can be manipulated, by partial denaturation, to adopt a conformation thought to resemble that of latent PAI-1 (Carrell et al., 1991), PAI-1 is the only serpin known to spontaneously adopt this conformation in vivo. The active form of PAI-1 is stabilized by interaction with vitronectin, a major component of plasma and extracellular matrix. Vitronectin-bound PAI-1 has a $T_{1 / 2}$ approximately double that of free PAI-1 (Declerck et al., 1988). Latent PAI-1 is probably released rapidly from vitronectin and cleared from plasma. Thus, the ability to adopt the latent conformation may provide a mechanism for sharply restricting the localization of PAI-1 activity.

Three-dimensional structure data are available for several members of the serpin family in varying conformations, beginning with the proteolytically cleaved form of $\alpha 1$-antitrypsin (Loebermann et al., 1984). In this latter structure, the $P_{1}$ and $P_{1}$, residues are located on opposite ends of the molecule, whereas in native $\alpha 1$-antitrypsin these residues are covalently linked. An intact loop, located above the plane of the molecule in an $\alpha$-helical configuration, was first identified in the structure of the non-inhibitory serpin, ovalbumin (Stein et al., 1990). The first solution for an uncleaved inhibitory serpin, latent PAI-1 (Mottonen et al., 1992), also contained an intact loop. However, it was fully inserted as the fourth strand of $\beta$-sheet $\mathrm{A}$, analogous to its location in cleaved serpins (Loebermann et al., 1984). Structures closely resembling ovalbumin have recently been reported for the presumed active form of antithrombin III (ATIII) and $\alpha 1$-antichymotrypsin (Carrell et al., 1994; Schreuder et al., 1994; Wei et al., 1994). These data support a proposed model for inhibitory serpin structure based on a mobile RCL projecting above the plane of the molecule (Carrell et al., 1991). Encounter with the protease is followed by loop insertion, locking inhibitor and protease into a stable, inactive complex (Lawrence et al., 1994b). Hydrolysis of the complex yields the cleaved form of the serpin, with the C-terminal portion of the loop fully inserted into $\beta$-sheet A.

In order to probe the biologic significance of the unique active to latent transition of PAI-1 and to further define 
the molecular basis of serpin function, we and others have previously used site-directed mutagenesis in an attempt to develop PAI-1 mutants with increased stability. Such rational protein engineering, based on accepted principles of serpin structure, have generally resulted in only modest increases in PAI-1 stability (Lawrence et al., 1994c; Tucker et al., 1994). We now report the application of functional screening to analyze a large library of recombinant PAI-1 variants constructed by random mutagenesis. Dramatic enhancement in PAI-1 functional stability was achieved by this approach, with increases in the $T_{1 / 2}$ of up to 72-fold. Detailed analysis of the contributing mutations demonstrates complex interactions between individual substitutions and provides new insights into the mechanism of PAI-1 latency.

\section{Results}

\section{Library construction and screening}

A library of random PAI-1 mutants with $>3 \times 10^{6}$ independent clones was constructed in the $\lambda$ phage expression vector $\lambda E X l o x$ by error-prone PCR. DNA sequence analysis of 34 individual subclones demonstrated a mutation frequency of $1: 461$, resulting in an average of approximately three amino acid substitutions per variant. Nucleotide substitutions were essentially random, though there was a slight excess of changes at A:T base pairs, similar to the pattern observed by others (Cadwell and Joyce, 1992). A higher mutation frequency was observed among mutants selected on the basis of increased stability (1:289), compared to randomly selected clones (1:461), or clones selected for wild-type inhibitory activity (1:669). Nearly half of the mutations observed in these latter clones were silent substitutions.

Recombinant PAI-1 mutants were screened for functional activity as phage plaques using a filter-based assay. This method takes advantage of the ability of PAI-1 to form SDS-stable complexes with tPA (Figure 1). With this assay, $\sim 25 \%$ of the phage plaques were observed to express functionally active PAI-1, consistent with previous observations in other proteins of the relative tolerance for amino acid substitution (Matsumura et al., 1988; Kleina and Miller, 1990). In order to identify mutant PAI-1s that are delayed in the transition from the active to latent forms, protein expression was induced and the phagecontaining plates were incubated for $12-18 \mathrm{~h}$ at $37^{\circ} \mathrm{C}$ (Figure 1). Under these conditions, wild-type (wt) PAI-1 would be expected to lose $>98 \%$ of its activity. Indeed, residual functional activity was detected in only a small subset of plaques, corresponding to $\sim 1: 7000$ mutant clones. Three hundred thousand phage were screened, and 15 clones which demonstrated functional activity after prolonged incubation were isolated, automatically subcloned and subjected to further analysis.

\section{Functional analysis of selected clones}

Functional stabilities at $37^{\circ} \mathrm{C}$ for wtPAI-1 and recombinant PAI-1 prepared from each of the selected clones are shown in Figure 2, with the $T_{1 / 2} \mathrm{~s}$ calculated from the curves listed in Table I. The $T_{1 / 2}$ observed for wtPAI-1 $(2 \mathrm{~h})$ is consistent with previous reports (Declerck et al., 1988; Lawrence et al., 1989, 1990, 1994c). All the selected

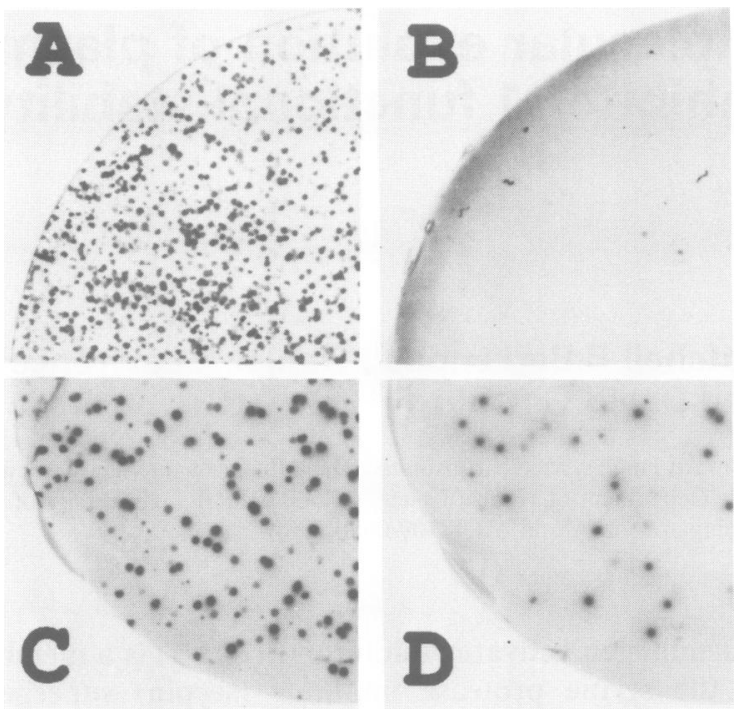

Fig. 1. A filter series showing immunoreactive plaques following a genetic screen for PAI-1 mutants with increased functional stability. Lawns of E.coli strain BL21 (DE3) pLys S infected with the PAI-1 random mutant $\lambda$ phage library (A and $\mathbf{B}$ ) or partially purified clone 19-1A (C and D) were reacted with tPA coated filters at the time of isopropylthio- $\beta$-D-galactoside induction $(A$ and $C)$ and again after overnight incubation at $37^{\circ} \mathrm{C}(\mathrm{B}$ and $\mathrm{D})$, and filters developed with $\alpha$ PAI-1 antibodies.

PAI-1 variants demonstrated increases in functional halflife, with $T_{1 / 2}$ s ranging from 5.6 to $145 \mathrm{~h}$.

Though the observed mutations dramatically increased functional stability, they did not appear to have major effects on other PAI-1 functions, such as PAI-1 inhibitory activity or vitronectin binding (not shown), suggesting only limited changes in overall PAI-1 structure. The single exception was clone 16-2 which displayed a significant decrease in vitronectin binding. This clone contains substitutions at both $G \ln 55$ and $G \ln 123$, sites which we have previously identified as part of the PAI-1 vitronectin binding domain (Lawrence et al., 1994a).

\section{DNA sequence analysis}

The entire coding region of all 15 clones selected for increased functional stability were subjected to DNA sequence analysis. Two clones contained the identical four nucleotide substitutions, suggesting that they were derived from the same original mutant in this amplified library. Of the remaining 14 clones, no two share more than one mutation. As shown in Table I, one to five mutations altering the amino acid sequence were identified in each clone, with a total of 35 distinct amino acid substitutions observed. At eight PAI-1 amino acid positions, the same or different substitutions were observed in two or more clones. Multiple occurrences of the same substitutions were observed at seven residues and in two instances, two different amino acid substitutions were detected at the same position (Phe372 to isoleucine or leucine, and Pro36 to serine or threonine).

\section{Analysis of individual PAI-1 point mutants}

The occurrence of at least one of the eight recurring mutations in all but one of the 14 selected clones (Table I) initially suggested that these eight mutations alone were responsible for the observed increased stability and that 


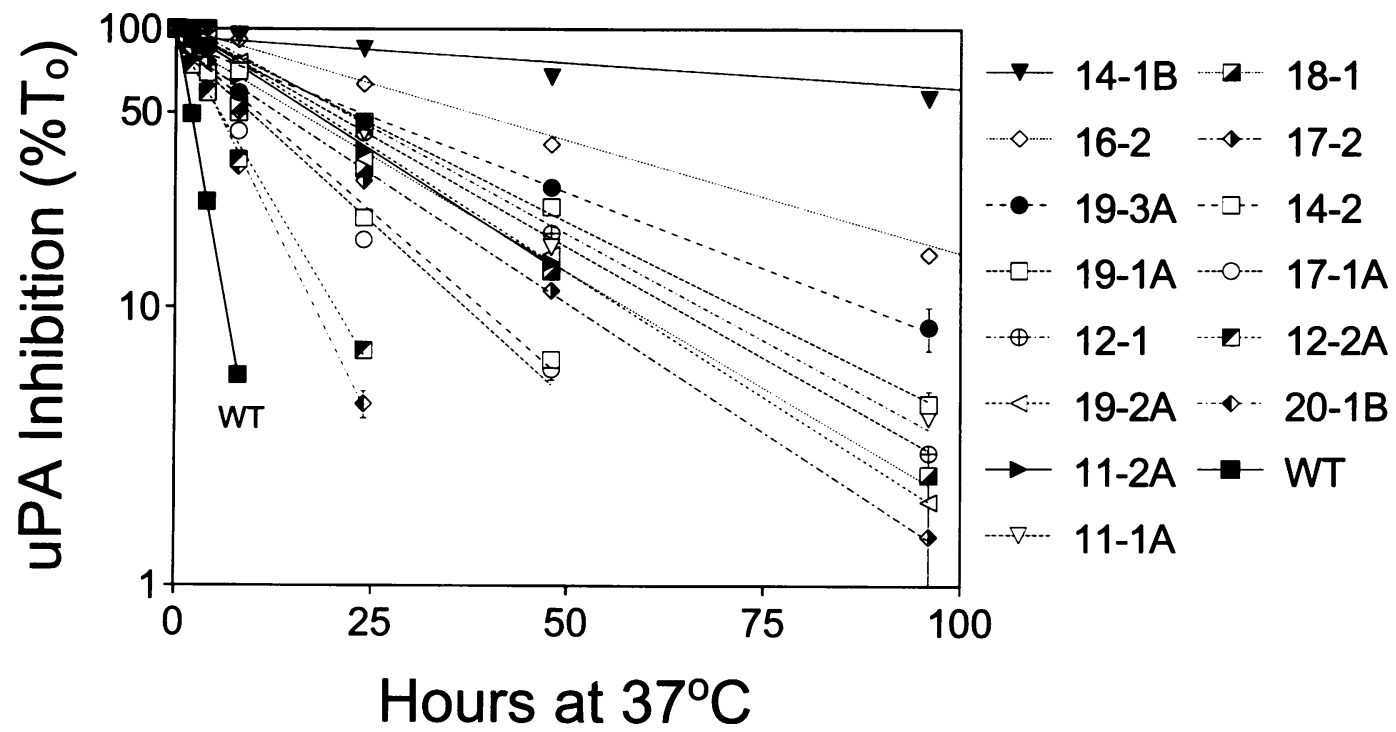

Fig. 2. Functional decay of inhibitory activity against uPA of wtPAI-1 and selected PAI-1 mutants. Residual uPA inhibitory activity is plotted, on a $\log$ scale, as a function of time at $37^{\circ} \mathrm{C}$. Each point represents the mean of at least two independent determinations $\pm \mathrm{SE}$. The $T_{1 / 2}$ of each clone, calculated from the formula of a fitted line using DeltaGraph Pro3 (DeltaPoint, Inc. Monterey, CA), is shown in Table I.

the other associated mutations were irrelevant products of random mutagenesis. To test this hypothesis, 18 of the 35 unique substitutions were expressed as isolated variants and analyzed for functional stability. The observed $T_{1 / 2} \mathrm{~S}$ are listed in Table II. With the exception of $\mathrm{N} 150 \mathrm{H}$, which actually decreased PAI-1 functional stability, seven of the eight recurrent mutations [P36(S,T), M45K, I91L, T93S, E327V, M354I, F372(I,L)] conferred an increased $T_{1 / 2}$. However, these effects were generally much less dramatic than those observed in the original compound mutants.

\section{Spatial location of the amino acid substitutions}

The locations of the 35 observed amino acid substitutions within the linear PAI-1 sequence are shown in Figure $3 \mathrm{~A}$. To evaluate the spatial relationships between these residues, a molecular model of the active PAI- 1 conformation was generated using the recently reported structure of uncleaved ATIII (Carrell et al., 1994). In Figure 3B, the positions of these variant amino acid residues within this model for active PAI- 1 are compared to their position within the known structure of the PAI-1 latent conformation (Mottonen et al., 1992). The mutations are observed to be distributed throughout the PAI-1 structure. Though there may be an over-representation of mutations within and around the RCL, no consistent pattern is evident to suggest a single, dominant molecular mechanism for the increased functional stability.

\section{Analysis of compound mutant 14-1B}

The most stable mutant, 14-1B, exhibited a $T_{1 / 2}$ of $145 \mathrm{~h}$ (Table I). However, each of the four mutations expressed individually on a wtPAI-1 background only resulted in a modest change in stability, with $T_{1 / 2} \mathrm{~s}$ ranging from 1.3 to $6.6 \mathrm{~h}$ (Table II). PAI-1 variants containing each possible combination of three mutations were constructed by sitedirected mutagenesis. As shown in Figure 4, the observed $T_{1 / 2} \mathrm{~S}$ range from $12.0 \mathrm{~h}$ to $91.4 \mathrm{~h}$, with none achieving the full stability of the original clone, containing all four mutations. Of note, the $\mathrm{N} 150 \mathrm{H}$ substitution is destabilizing when expressed as a single mutation, ( $T_{1 / 2}$ reduced to
$1.3 \mathrm{~h}$ ), or in combinations with M354I and Q319L (Figure 4). However, combining N150H with K154T leads to an approximate doubling of $T_{1 / 2}$.

Recombinant PAI-1 mutant 14-1B was expressed in E.coli, purified, and subjected to further analysis. The 14$1 \mathrm{~B}$ protein retained vitronectin binding function, as noted above, and also eluted from heparin Sepharose with a profile indistinguishable from that of wtPAI-1, indicating that the variant molecule also maintains normal affinity for this ligand (Lawrence et al., 1989). Purified 14-1B was subjected to thermal denaturation analysis with the results shown in Figure 5. A markedly increased thermal stability of the 14-1B active form relative to active wtPAI-1 was observed, with a dramatic rise in melting temperature $\left(T_{\mathrm{M}}\right)$ of nearly $8^{\circ} \mathrm{C}$ to $57^{\circ} \mathrm{C}$, similar to the reported $T_{\mathrm{M}} \mathrm{S}$ of other native serpins (Schulze et al., 1994).

To examine the interaction of mutant 14-1B with uPA and tPA, SDS-PAGE analysis of complex formation was performed (Figure 6). At equimolar concentrations of inhibitor and protease, nearly all of the mutant 14-1B could be seen to form a 1:1 SDS-stable complex with either uPA or tPA. A small amount of 14-1B appeared to be cleaved following reaction with tPA, $\sim 3$-fold more than with uPA (Figure 6), and notably increased compared to wtPAI-1 (not shown). This slightly enhanced substrate behavior with IPA may result from a reduced rate of RCL insertion following reaction with tPA, as we have observed with other PAI-1 mutants (Lawrence et al., 1994b). An additional minor band at $\sim 33 \mathrm{kDa}$ is also observed following interaction of tPA (lane 4), probably representing cleavage at another site outside of the RCL. A much fainter band at the same position is also seen with uPA (lane 2). The rates of association of mutant 14-1B and PAs were also determined as described (Lawrence et al., 1990) and compared with values obtained for wtPAI-1. Similar to other PAI-1 mutants (Lawrence et al., 1994a,b), the second-order rate constant $\left(k_{\mathrm{i}}\right)$ for mutant 14-1B against uPA was nearly identical to wtPAI- $1,[(8.8 \pm$ $0.6) \times 10^{6}$ versus $\left.(1.4 \pm 0.1) \times 10^{7} / \mathrm{M} / \mathrm{s}\right]$. However, against tPA, the second-order rate for mutant $14-1 B$ was 
Table I. Observed amino acid substitutions and half-lives of 14 independent PAI-1 mutant clones, selected for increased functional stability

\begin{tabular}{|c|c|c|c|c|c|c|}
\hline \multirow{2}{*}{$\frac{\text { Clone }}{11-2 \mathrm{~A}}$} & \multicolumn{5}{|c|}{ Amino acid substitution $^{\mathrm{a}}$} & \multirow{2}{*}{$\frac{T_{1 / 2}(\mathrm{~h})^{\mathrm{b}}}{17.4( \pm 0.6)}$} \\
\hline & P36S & $\mathrm{T} 49 \mathrm{~A}$ & \multirow{3}{*}{ K141Q } & & & \\
\hline $12-1$ & P36T & $\mathrm{A} 12 \mathrm{~V}$ & & & & $21.5( \pm 0.2)$ \\
\hline $11-1 A$ & & $191 \mathrm{~L}$ & & & & $18.4( \pm 1.1)$ \\
\hline $19-2 \mathrm{~A}$ & & $191 \mathrm{~L}$ & \multirow{2}{*}{$\begin{array}{l}\text { T93S } \\
\text { T93S }\end{array}$} & & & $18.1( \pm 0.2)$ \\
\hline $19-3 A$ & A318S & V334E & & \multirow{2}{*}{$\begin{array}{l}\text { M354I } \\
\text { M354I }\end{array}$} & & $23.5( \pm 0.7)$ \\
\hline $14-1 \mathrm{~B}$ & K154T & Q319L & & & \multirow{2}{*}{$\begin{array}{l}\mathrm{N} 150 \mathrm{H} \\
\mathrm{N} 150 \mathrm{H}\end{array}$} & $145.4( \pm 5.3)$ \\
\hline $16-2$ & M45K & Q55L & Q123R & T333S & & $37.9( \pm 1.2)$ \\
\hline $14-2$ & M45K & G84R & F236I & & \multirow{7}{*}{ T339S } & $10.4( \pm 0.2)$ \\
\hline $12-2 \mathrm{~A}$ & M45K & E212V & A156S & V324A & & $5.6( \pm 0.0)$ \\
\hline $17-2$ & L160Q & M235L & E327V & V343A & & $12.9( \pm 0.5)$ \\
\hline $19-1 \mathrm{~A}$ & $\mathrm{~T} 232 \mathrm{~S}$ & & E327V & F372I & & $22.0( \pm 0.2)$ \\
\hline $20-1 B$ & & & & F372I & & $5.6( \pm 0.2)$ \\
\hline $18-1$ & $\mathrm{I} 253 \mathrm{~T}$ & $\mathrm{R} 271 \mathrm{C}$ & & F372L & & $15.6( \pm 0.7)$ \\
\hline $17-1 \mathrm{~A}$ & S331G & S13K & $\mathrm{D} 14 \mathrm{~T}$ & & & $9.5( \pm 0.5)$ \\
\hline
\end{tabular}

${ }^{\text {a} B o x e s ~ i n d i c a t e ~ r e c u r r e n t ~ m u t a t i o n s . ~}$

${ }^{\mathrm{b}} T_{1 / 2} \mathrm{~s}$ are taken from the data shown in Figure 2.

reduced $\sim 10$-fold compared with wtPAI- $1,[(2.2 \pm 0.1) \times$ $10^{6}$ versus $\left.(2.3 \pm 0.1) \times 10^{7} / \mathrm{M} / \mathrm{s}\right]$. This latter result could be related to the enhanced substrate behavior of this mutant toward tPA, described above.

\section{Discussion}

Though it is frequently possible to alter function by sitedirected mutagenesis, it has generally proven difficult to enhance the natural activity of a protein beyond that of the wild-type molecule. We recently generated the full range of reactive center $P_{1}-P_{1^{\prime}}$ substitutions in PAI-1 and obtained a number of variants with altered target protease specificity. However, none of these variants exhibited a rate of inhibition against IPA or uPA that was greater than that of wtPAI-1 (Sherman et al., 1992, 1995). Rational approaches to increase protein stability based on structural modeling have also generally met with limited success (Matthews, 1987; Lawrence et al., 1994c). Previous random mutagenesis approaches aimed at enhancing protein function have focused on prokaryotic proteins and the degrees of increased stability which have been achieved have usually been modest. It is generally assumed that evolution has fine-tuned each protein for optimum function. For this reason, random mutagenesis experiments probing protein function frequently begin with a crippled molecule that already contains a negative mutation and select for amino acid substitutions which suppress this defect and result in reversion of protein function to (or near), that of the original wild-type molecule (Matthews, 1987; Knowles, 1991; Mitraki et al., 1991; Kwon et al., 1994). Similarly, a number of investigators have selected for mutations that adapt a protein to function under altered conditions, such as increasing organic solvent concentrations or elevated temperatures (Chen and Arnold, 1993; Flam, 1994; Kwon et al., 1994). Perhaps the most striking example of molecular selection to engineer
Table II. Functional half-lives of individual site-directed PAI-1 mutants

\begin{tabular}{ll}
\hline Mutation & $T_{1 / 2}(\mathrm{~h})^{\mathrm{a}}$ \\
\hline Wild-type & $2.0( \pm 0.04)$ \\
P36S & $4.9( \pm 0.42)$ \\
P36T & $6.5( \pm 0.27)$ \\
M45K & $4.1( \pm 0.06)$ \\
T49A & $2.4( \pm 0.02)$ \\
191L & $18.4( \pm 1.07)$ \\
T93S & $3.2( \pm 0.03)$ \\
K141Q & $1.4( \pm 0.02)$ \\
N150H & $1.3( \pm 0.02)$ \\
K154T & $3.4( \pm 0.06)$ \\
E212V & $1.9( \pm 0.07)$ \\
T232S & $2.3( \pm 0.03)$ \\
R271C & $5.4( \pm 0.11)$ \\
Q319L & $2.9( \pm 0.07)$ \\
E327V & $6.0( \pm 0.34)$ \\
S331G & $11.6( \pm 0.35)$ \\
M354I & $6.6( \pm 0.40)$ \\
F372I & $5.6( \pm 0.22)$ \\
F372L & $3.6( \pm 0.04)$ \\
\hline
\end{tabular}

${ }^{a}$ Half-lives were calculated as described in Materials and methods. The data represent the average of at least two separate determinations with each of two clones \pm SE.

enhanced function of a native protein was recently demonstrated for the bacterial enzyme $\beta$-lactamase (Stemmer, 1994). The current report is the first successful application of a similar random mutagenesis approach to enhance the function of a mammalian protein.

\section{Large increases in PAl-1 stability require contributions from multiple amino acid substitutions}

Given a total of $\sim 7600$ possible single amino acid substitutions in the 379 residue mature PAI-1 coding sequence $(379 \times 19)$, and assuming a Poisson distribution of 
A

1 VHHPPSYVAH LASDFGVRVF QQVAQASKDR NVVFSPYGVA SVLATूLQLIT $h C$

$h D$

$s 2 A$

51 GGETQQQIQA AMGFKIDDKG MAPALRHLYK ELMGPWNKDE ISTTTDAFVQ $h E \quad S I A \quad h F$

101 RDLKLVQGFM PHFFRLFRST VKOVDFSEVE RARFIINDWV KTHTKGMISTS

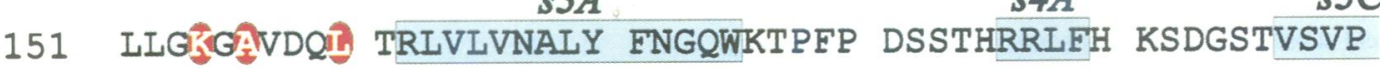

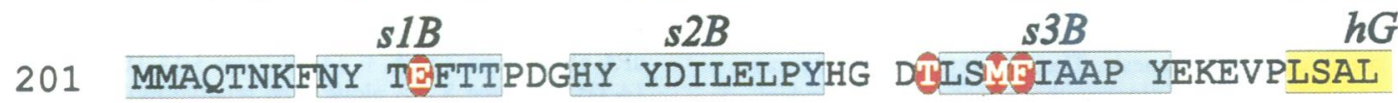

$\begin{array}{cccc}h H & s 2 C & s 6 A & h I \\ & \text { TNILSAQLIS HWKGNMTRLP RLLVLPKFSL ETEVDLRKPL ENLGMTDMFR }\end{array}$

$s 5 A \quad R C L(s 4 A)$

301 QFQADFTSLS DQEPLHVAOQA LQKVKIÊVNE SGTVASSSTA VIVASARMAPE $s 1 C \quad s 4 B \quad s 5 B$

351 EITIEDRPFLF VVRHNPTGTV LGMMGQVMEP

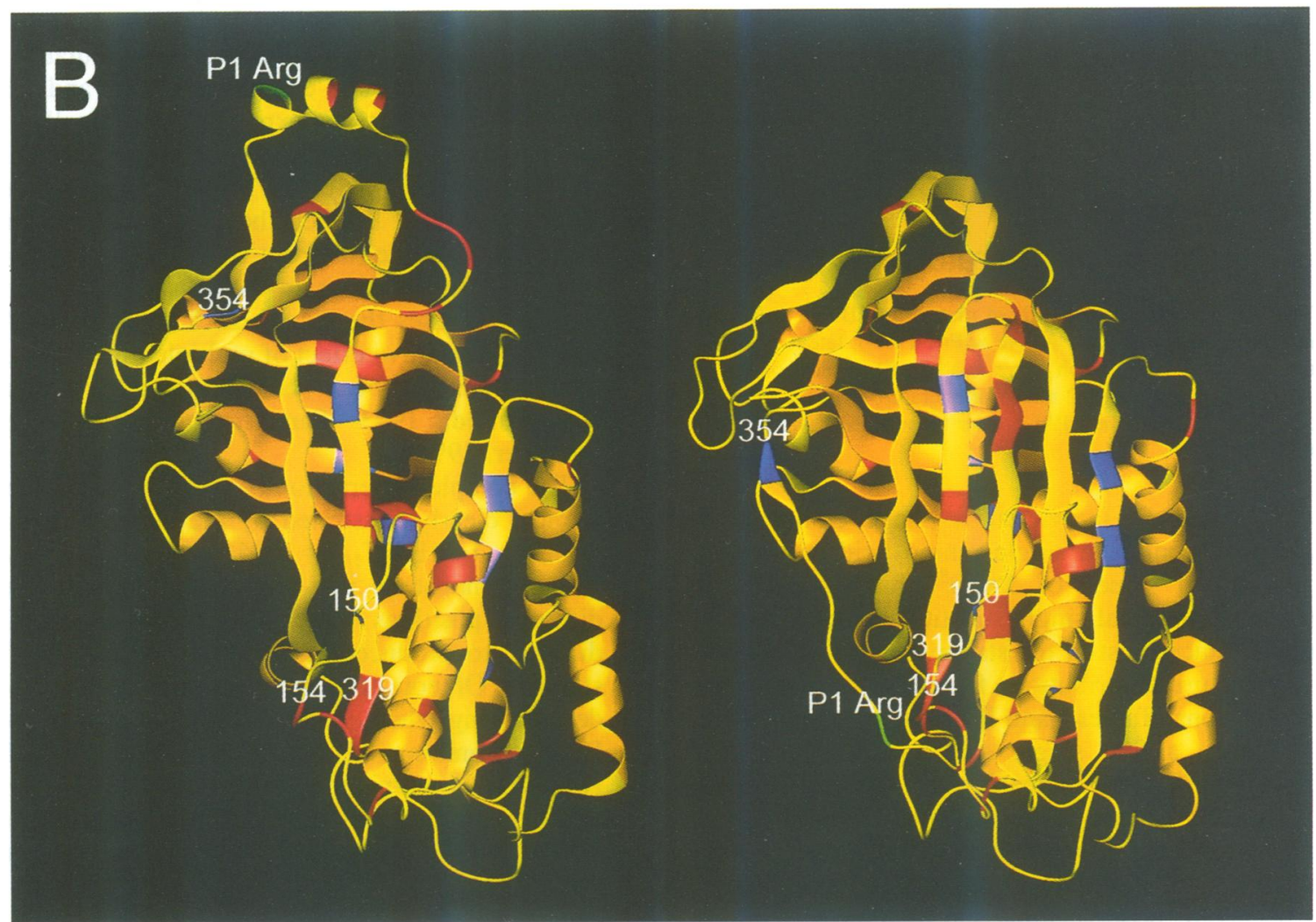

Fig. 3. Placement of the 35 amino acid substitutions within the PAI-1 primary and tertiary structures. (A) Linear amino acid sequence of mature PAI- 1 with the $\alpha$-helices indicated in yellow blocks and $\beta$-strands indicated in blue. Individual $\alpha$-helices are labeled above each yellow block (hA-hI). Individual strands (1-6) in each $\beta$-sheet (A-C) are also labeled (e.g. s6A is strand 6 in $\beta$-sheet A). The reactive center loop (RCL) is also labeled (s4A). The positions of the eight recurrent mutations are shown as dark blue circles, the 25 unique mutations in red, and the $P_{1}$ arginine in green. (B) Ribbon diagram of the active (left) and latent (right) PAI-1 structures. Mutation positions are indicated with the same colors as described for (A). The residue numbers for the four mutations present in clone 14-1B are labeled in each ribbon structure (N150H, K154T, Q319L, and M354I). 


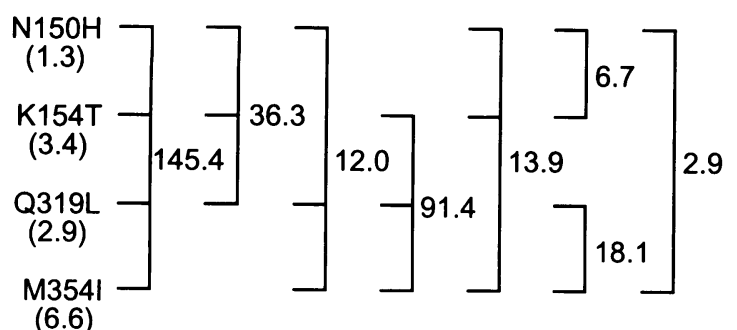

Fig. 4. Functional half-lives of PAI-1 mutants containing the indicated combinations of amino acid substitutions. Stabilities of each individual mutation, taken from Table II, are shown in the left column under each amino acid substitution, as $T_{1 / 2}$ in hours. Each tested combination is indicated by a bracket, with the observed $T_{1 / 2}$ to the right. PAI-1s containing each indicated combination of amino acid substitution were generated by site-directed mutagenesis. The data represent the average of at least two separate determinations with each of two independent clones. All standard errors were $<5 \%$.

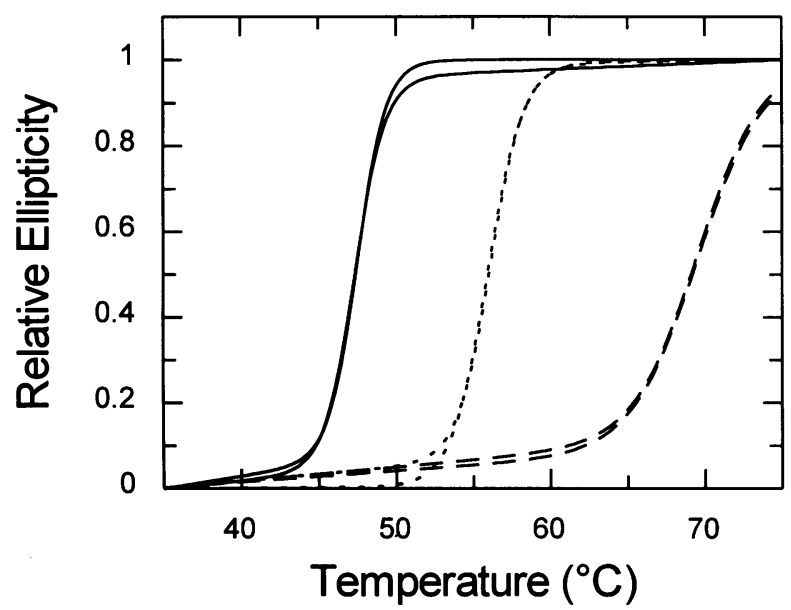

Fig. 5. Thermal denaturation analysis of mutant 14-1B and wtPAI-1 in active and latent conformations. Active wtPAI-1 (-), latent wtPAI-1 $(---)$, mutant 14-1B $(\cdots)$. Each curve is a best-fit of plots of the relative ellipticity change at $222 \mathrm{~nm}$ vs temperature over the range of 35 to $75^{\circ} \mathrm{C}$. The data for latent PAI-1 were taken from Lawrence et al. (1994b).

mutations, $\sim 20 \%$ of individual $\lambda$ phage clones in the library should contain a single amino acid substitution. Thus, the initial screen of $3 \times 10^{5}$ phage should have analyzed each potential single substitution approximately eight times. Since most of the stable mutants identified in this study require contributions from two or more amino acid substitutions (Tables I and II), these data suggest that there are only a few single amino acid substitutions capable of significantly increasing the stability of native PAI-1. Thus, it is the power of combinatorial genetic screening which made possible the identification of the dramatically stabilized PAI-1 variants reported here. It should be noted, however, that the qualitative nature of the functional screen most likely introduced a bias toward the most stable mutants. It is likely that variants with less than a 3-fold increase in $T_{1 / 2}$, such as the set of sitedirected mutants previously identified by our group (Lawrence et al., 1994c) and most of the single mutants described here (Table II), would have been missed.

Though 12 of the 14 stable PAI-1 variants identified in the genetic screen contain multiple amino acid substitutions (Table I), a subset of these mutations appear to be incidental, with no significant contribution to stability.

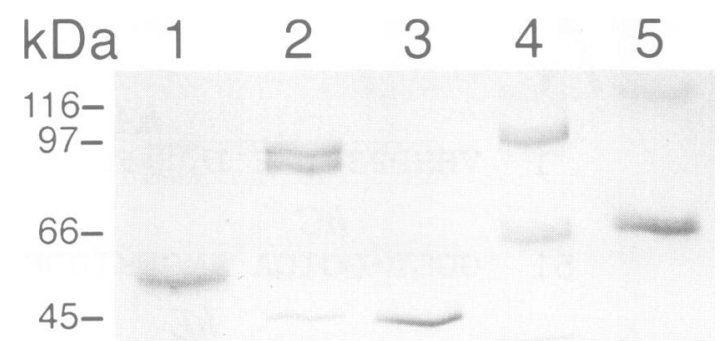

29-

Fig. 6. SDS-PAGE analysis of PAI-1 14-1B \pm uPA or tPA. Lane 1 uPA alone, lane $2 \mathrm{uPA}+14-1 \mathrm{~B}$, lane $314-1 \mathrm{~B}$ alone, lane $4 \mathrm{tPA}+$ 14-1B, and lane 5 tPA alone. The relative mobilities of protein standards are indicated on the left.

Specifically, though variants 12-2A, 17-1A, and 19-2A contain two to five substitutions, the increased functional stability of each mutant can be ascribed to a different single substitutions (M45K, S331G and $191 \mathrm{~L}$, respectively, Tables I and II).

For the remaining nine clones, contributions from more than one amino acid position appear to be required for maximal, functional stability. In some instances, individual substitutions appear to be simply cumulative in their stabilizing affect. For example, E327V and F372I both have $T_{1 / 2}$ of $\sim 6 \mathrm{~h}$, when expressed individually, increasing to $22 \mathrm{~h}$ when they are expressed in cis. Similarly, M354I and T93S, with $T_{1 / 2}$ s of 6.6 and $3.2 \mathrm{~h}$ respectively, result in a $T_{1 / 2}$ of $12.2 \mathrm{~h}$ when they are expressed together in the same molecule (data not shown). The presence of two additional substitutions in the compound mutant 19-3A, further increases the $T_{1 / 2}$ to $23.5 \mathrm{~h}$ (Table I). A similar, seemingly additive effect of multiple mutations on protein function has also been observed for other proteins (Chen and Arnold, 1993; Sandberg and Terwilliger, 1993; Stemmer, 1994).

The complex interaction between multiple substitutions is most clearly illustrated by the clone with the highest stability, 14-1B $\left(T_{1 / 2}=145 \mathrm{~h}\right)$. The results discussed above demonstrate that amino acid substitutions at four different positions must interact, either directly or indirectly, to produce the maximal increase in functional stability. Of note, one of these substitutions, $\mathrm{N} 150 \mathrm{H}$, is destabilizing when expressed as a single mutation (Table II) or in combinations with M354I and Q319L (Figure 3). However, combining $\mathrm{N} 150 \mathrm{H}$ with $\mathrm{K} 154 \mathrm{~T}$ leads to an approximate doubling of $T_{1 / 2}$. These observations suggest a complex, local structural interaction between $\mathrm{N} 150 \mathrm{H}$ and K154T.

\section{The structural basis for increased PAl-1 functional stability}

Based on current models of serpin function (Carrell et al., 1991; Lawrence et al., 1994b; Potempa et al., 1994; Wei et al., 1994; Stein and Carrell, 1995), amino acid substitutions that stabilize active PAI-1 might be expected to cluster within the RCL or in the strands of $\beta$-sheet A adjacent to the site of RCL insertion. Indeed, at least one of the mutations located in the RCL, S331G, significantly increases PAI-1 functional stability. This mutation occurs 
in the reverse-turn between strands $5 \mathrm{~A}$ and $4 \mathrm{~A}$ and would likely be the first residue to move into sheet $\mathrm{A}$ during the active to latent transition (Figure 3 and Carrell et al., 1991; Mottonen et al., 1992; Stein and Carrell, 1995). Though several other mutations are also located in the vicinity of the RCL, most of the 35 substitutions identified in the functionally stable variants appear to be randomly scattered throughout the PAI-1 structure (Figure 3A). These data suggest that the observed PAI-1 functional stabilization does not result simply from direct steric effects on RCL insertion into $\beta$-sheet $A$, and must involve other, more complex structural mechanisms. Consistent with this hypothesis, previous studies replacing the RCL of PAI- 1 with the RCL of other stable serpins demonstrated no difference in the rate of PAI-1 conversion to the latent conformation (Lawrence et al., 1990).

In previous work (Lawrence et al., 1994c), we engineered PAI-1 variants with modest increases in functional stability by site-directed mutagenesis (approximate doubling of the $T_{1 / 2}$ ). Thermal denaturation studies indicated that these changes were associated with significant destabilization of the latent form, with little or no effect on the active form. In contrast to these earlier variants, thermal denaturation analysis of the 14-1B protein demonstrates markedly increased thermal stability of the active form relative to active wtPAI-1, with a rise in $T_{\mathrm{M}}$ of nearly $8^{\circ} \mathrm{C}$ to $57^{\circ} \mathrm{C}$ (Figure 5), similar to the reported $T_{\mathrm{M}}$ s of other native serpins (Schulze et al., 1994). These data suggest that the increased functional stability of PAI-1 mutant 14-1B is due, at least in part, to structural stabilization of the active form, decreasing the thermodynamic drive toward the more stable latent structure. This structural stabilization need not necessarily involve the RCL or affect its mechanism of insertion, but instead may occur through subtle optimization of hydrophobic interactions and more compact folding of the PAI-1 molecule. Indeed, results of studies with other proteins have generally supported the notion that thermodynamic stabilization of a protein is global and that the contribution of independent mutations can be at least qualitatively additive (Matthews, 1987; Sandberg and Terwilliger, 1993; Stemmer, 1994), as we have observed here. Crystallographic studies of site-directed mutations in other proteins have generally demonstrated only small, localized alterations in 3-dimensional structure, with movement of side chain atoms in the vicinity of the substitution only in the order of $1 \AA$, and adjustments of the backbone of even smaller magnitude (Matthews, 1987). Studies of mutant T4 lysozymes with multiple substitutions at one position (Ile3) demonstrated a direct relationship between the hydrophobicity of the substituted residue and the resulting increase in stability (Matsumura et al., 1988).

\section{Amino acid substitutions at conserved positions}

Though mutations at highly conserved amino acid positions often results in loss of protein function, several of the substitutions observed here are notable exceptions. Pro36 and Phe372 (located in $\alpha$-helix B and strand 5 of $\beta$-sheet $B$, respectively), are nearly invariant among known serpins, including PAI-1. However, substitution of either isoleucine or leucine for Phe372, and either serine or threonine for Pro36 not only fail to disrupt PAI-1 stability, but actually markedly enhance it (Table II). In contrast, a proline to threonine substitution in ATIII (P80T), the position homologous to Pro36 in PAI-1, is associated with a type I deficiency state manifesting reduced synthesis of ATIII (Millar et al., 1994). This conserved proline is thought to initiate formation of $\alpha$-helix B. No structural explanation is readily apparent for these paradoxical results involving similar substitutions in these two members of the serpin gene family.

I91L, the most stable single amino acid substitution in our study and the only single mutant identified in the library screen (Table I), also occurs at a highly conserved amino acid residue. Nearly all known inhibitory serpins contain either a leucine or valine residue (usually the former) at this position, which is located at the N-terminal end of strand 2 in $\beta$-sheet $A$. Non-inhibitory serpins, such as ovalbumin, corticosteroid binding globulin, and angiotensinogen, have divergent substitutions at this position (phenylalanine, methionine, and glutamine). Isoleucine at this position is unique to PAI-1, and is present in all four mammalian species for which sequence data is available. Thus, the most stabilizing single amino acid substitution we have identified in PAI- 1 represents the substitution of the serpin consensus residue at a singularly non-conservative $\mathrm{PAI}-1$ position. At least five other substitutions identified in the 14 stable clones (Table I) also represent mutations at uniquely non-conserved positions in PAI-1 (T49A, R271C, A318S, V324A, V334E), though only the latter three examples clearly bring the variant closer to the other serpins. These observations suggest that the divergence of PAI-1 from the serpin consensus sequence at these positions may be associated with its unique ability to adopt the latent conformation and that mutation back to the consensus alters PAI-1 toward the more stable, active structure characteristic of other serpins. This association of divergent residues in PAI-1 with decreased functional stability suggests that the unique latent form of PAI-1 may have resulted from positive evolutionary selection.

\section{Evolution of the labile PAI-1 structure}

The results reported here demonstrate that it is possible to dissociate the inherent instability of native PAI-1 from its functional activity. This contrasts with recent observations in T4 lysozyme, suggesting that nature often sacrifices stability to achieve optimum function (Shoichet et al., 1995). The dramatic increase in PAI-1 stability which can be engineered by random mutagenesis, compared to the more modest changes generally observed with other native proteins (Sandberg and Terwilliger, 1993; Kwon et al., 1994; Stein and Carrell, 1995), suggests that PAI-1 has not been selected by evolution for maximal stability under physiologic conditions, a notion which on first glance would appear to be non-adaptive. The association of PAI-1 residues which diverge from the serpin consensus sequence with decreased functional stability further suggests that the unique PAI-1 latent form may have resulted from positive evolutionary selection. Perhaps the tight interaction between PAI- 1 and its stabilizing co-factor vitronectin may have removed the pressure to conserve the stable conformation, which applies to other members of the serpin family lacking such a co-factor. Alternatively, stable expression of PAI- 1 activity in one or more biologic compartments could have deleterious consequences, 
driving selection for the latent PAI-1 conformation as a regulatory mechanism. It is interesting to note that mice null for PAI-1 are protected against the thrombotic reaction induced by endotoxin (Carmeliet et al., 1993). The decreased thermal stability of wtPAI- 1 could accelerate loss of activity during inflammatory processes associated with fever, thereby decreasing the risk of pathologic thrombosis. The highly stable PAI-1 variants identified in this study provide a powerful new tool for probing the physiological significance of PAI-1 latency in vivo.

\section{Material and methods}

\section{Construction and screening of random mutant library}

The entire coding sequence of mature PAI-1 was amplified by four cycles of error-prone PCR, as previously described (Lawrence $e t$ al., 1994a). The mutagenized product was gel purified from the template and re-amplified using standard PCR conditions to produce an amplified pool of randomly mutagenized PAI-1 cDNAs. The PCR product was restricted with $X b a \mathrm{I}$ and $E c o$ RI, ligated to similarly restricted $\lambda$ EXlox arms (Novagen), and packaged with PhagePrep (Novagen). Lawns of phage-infected Escherichia coli strain BL21(DE3) pLys S were prepared and PAI-1 expression was induced, essentially according to manufacturer's instructions (Novagen), by incubation for $2 \mathrm{~h}$ at $37^{\circ} \mathrm{C}$ with nitrocellulose filters that had been soaked in isopropylthio- $\beta$-D-galactoside $(10 \mathrm{mM})$ and tPA $(10 \mu \mathrm{g} / \mathrm{ml}$ Activase, Genetech, San Francisco, CA) and blocked with $1 \%$ BSA/5\% milk (Carnation). Following removal of the filters, the phage containing plates were incubated overnight at $37^{\circ} \mathrm{C}$ then rescreened with a second tPA-coated filter for $2 \mathrm{~h}$. All filters were washed for $30 \mathrm{~min}$ in TBS containing $0.5 \%$ SDS and filter-bound PAI- 1 was detected by reaction with biotin-labeled $\alpha$ PAI- 1 antibodies (Sherman et al., 1992) and streptavidin-conjugated alkaline phosphatase (Gibco BRL).

\section{Site-directed mutagenesis}

Individual PAI-1 site-directed mutants were constructed using the Altered Sites mutagenesis kit (Promega), as described (Lawrence et al., 1994c) and sequenced throughout the mutagenized region. Although the possibility of an additional mutation, outside the mutagenized segments, cannot be completely excluded, we have previously observed a very low rate of non-targeted mutations with this procedure $(<1$ mutation/ $3.5 \times 10^{4} \mathrm{bp}$ ) (Lawrence et al., 1994b). In addition, E.coli lysates for each PAI-1 site-directed mutant were prepared and analyzed from two independent clones, as described (Lawrence et al., 1994a). Thus, effects on PAI-1 functional stability from unexpected mutations that arose during site-directed mutagenesis are extremely unlikely.

\section{Determination of the functional half lives $\left(T_{1 / 2}\right)$ at $37^{\circ} \mathrm{C}$}

PAI-1 cDNA mutants from purified plaques were automatically subcloned using the Cre/loxP system (Novagen), expressed in E.coli strain BL21(DE3) pLys S, and lysates prepared as previously described (Lawrence et al., 1994c). An aliquot of wild-type PAI-1 and each of the subcloned mutants, diluted 1:10 in $150 \mathrm{mM} \mathrm{NaCl}, 50 \mathrm{mM}$ Tris $\mathrm{pH} 7.5$, $100 \mu \mathrm{g} / \mathrm{ml} \mathrm{BSA}$, was analyzed for functional stability as measured by inhibitory activity against uPA (high molecular weight two-chain uPA, $3000 \mathrm{IU} / \mathrm{mL}$, American Diagnostica, Greenwich, CT) using the single step chromogenic substrate assay as described (Lawrence et al., 1989). The inhibitory activity against uPA remaining in each sample was measured following the indicated times of incubation at $37^{\circ} \mathrm{C}$ and normalized to the $100 \%$ value at time zero.

\section{Model of active PAI-1}

The model of active PAI-1 was generated in Quanta (Burlington, MA) by superposition of latent PAI-1 secondary structures on the tertiary structure of the active form of ATIII. The RCL structure $\left(\mathrm{P}_{16}-\mathrm{P}_{5^{\prime}}\right)$ was modeled using co-ordinates from ovalbumin. The $\mathrm{C} \alpha$ trace of the model, excluding the RCL, had a root mean square difference of $2.32 \AA$ and $1.43 \AA$ with latent PAI-1 and active ATIII, respectively. The atomic coordinates of ATIII were generously supplied by Drs R.Carrell and P.Stein (Carrell et al., 1994), and latent PAI-1 by Dr E.Goldsmith (Mottonen et al., 1992). The ovalbumin co-ordinates (Stein et al., 1990) were obtained from the Brookhaven database.

\section{Thermal denaturation analysis}

Mutant 14-1B and wtPAI-1 were purified as described (Lawrence et al., 1989). Duplicate PAI-1 samples were diluted to $40 \mu \mathrm{g} / \mathrm{ml}$ and analyzed on a JASCO J-710 spectropolarimeter as previously described (Lawrence et al., 1994c).

\section{PA-PAI-1 complex formation}

$1 \mu \mathrm{M}$ of purified mutant $14-1 \mathrm{~B}$ was incubated in $50 \mathrm{mM}$ Tris $\mathrm{pH} 7.8$, $100 \mathrm{mM} \mathrm{NaCl}$ for $30 \mathrm{~min}$. at $23^{\circ} \mathrm{C}$ alone or with $1 \mu \mathrm{M}$ uPA or tPA followed by addition of SDS to $1 \% .4 \mu$ l samples were then separated by SDS-PAGE on a $12.5 \%$ homogeneous gel (Phastgel, Pharmacia), followed by staining with Coomassie Blue.

\section{Acknowledgements}

We thank R.Kaufman, J.Lowe, G.Nable and S.Olson for helpful discussions, S.Labun for assistance in preparation of the manuscript, and the University of Michigan Biomedical Research Core Facilities for use of the spectropolarimeter. This work was supported in part by National Institutes of Health grant HL49184. D.G. is a Howard Hughes Medical Institute Investigator.

\section{References}

Cadwell,R.C. and Joyce,G.F. (1992) Randomization of genes by PCR mutagenesis. PCR Methods and Applications, 2, 28-33.

Carmeliet,P., Stassen,J.M., Schoonjans,L., Ream,B., van den Oord,J.J., De Mol,M., Mulligan,R.C. and Collen,D. (1993) Plasminogen activator inhibitor-1 gene-deficient mice. II. Effects on hemostasis, thrombosis, and thrombolysis. J. Clin. Invest., 92, 2756-2760.

Carmeliet,P., Schoonjans,L., Kieckens,L., Ream,B., Degen,J., Bronson,R., De Vos,R., van den Oord,J.J., Collen,D. and Mulligan,R.C. (1994) Physiological consequences of loss of plasminogen activator gene function in mice. Nature, 368, 419-424.

Carrell,R.W., Evans,D.L. and Stein,P.E. (1991) Mobile reactive center of serpins and the control of thrombosis. Nature, 353, 576-578.

Carrell,R.W., Stein,P.E., Fermi,G. and Wardell,M.R. (1994) Biological implications of a $3 \AA$ structure of dimeric antithrombin. Structure, 2 , 257-270.

Chen,K. and Arnold,F.H. (1993) Tuning the activity of an enzyme for unusual environments: Sequential random mutagenesis of subtilisin $\mathrm{E}$ for catalysis in dimethylformamide. Proc. Natl Acad. Sci. USA, 90, $5618-5622$.

Declerck,P.J., De Mol,M., Alessi,M.C., Baudner,S., Pâques,E.-P., Preissner,K.T., Müller-Berghaus,G. and Collen,D. (1988) Purification and characterization of a plasminogen activator inhibitor 1 binding protein from human plasma. J. Biol. Chem., 263, 15454-15461.

Fay,W.P., Shapiro,A.D., Shih,J.L., Schleef,R.R. and Ginsburg,D. (1992) Complete deficiency of plasminogen-activator inhibitor type 1 due to a frame-shift mutation. N. Engl. J. Med., 327, 1729-1733.

Flam,F. (1994) Co-opting a blind watchmaker. Science, 265, 1032-1033.

Kleina,L.G. and Miller,J.H. (1990) Genetic studies of the lac repressor XIII. Extensive amino acid replacements generated by the use of natural and synthetic nonsense suppressors. J. Mol. Biol., 212, 295-318.

Knowles,J.R. (1991) Enzyme catalysis: not different, just better. Nature, 350, 121-124.

Kwon,K.-S., Kim,J., Shin,H.S. and Yu,M.-H. (1994) Single amino acid substitutions of $\alpha_{1}$-antitrypsin that confer enhancement in thermal stability. J. Biol. Chem., 269, 9627-9631.

Lawrence,D., Strandberg,L., Grundström,T. and Ny,T. (1989) Purification of active human plasminogen activator inhibitor 1 from Escherichia coli. Comparison with natural and recombinant forms purified from eucaryotic cells. Eur. J. Biochem., 186, 523-533.

Lawrence,D.A., Strandberg,L., Ericson,J. and Ny,T. (1990) Structurefunction studies of the SERPIN plasminogen activator inhibitor type 1: analysis of chimeric strained loop mutants. J. Biol. Chem., 265, 20293-20301.

Lawrence,D.A., Berkenpas,M.B., Palaniappan,S. and Ginsburg,D. (1994a) Localization of vitronectin binding domain in plasminogen activator inhibitor-1. J Biol. Chem., 269, 15223-15228.

Lawrence,D.A., Olson,S.T., Palaniappan,S. and Ginsburg,D. (1994b) Serpin reactive-center loop mobility is required for inhibitor function but not for enzyme recognition. J. Biol. Chem., 269, 27657-27662.

Lawrence,D.A., Olson,S.T., Palaniappan,S. and Ginsburg,D. (1994c) 
Engineering plasminogen activator inhibitor-1 (PAI-1) mutants with increased functional stability. Biochemistry, 33, 3643-3648.

Loebermann,H., Tokuoka,R., Deisenhofer,J. and Huber,R. (1984) Human $\alpha_{1}$-proteinase inhibitor. Crystal structure analysis of two crystal modifications, molecular model and preliminary analysis of the implications for function. J. Mol. Biol., 177, 531-557.

Matsumura,M., Becktel,W.J. and Matthews,B.W. (1988) Hydrophobic stabilization in T4 lysozyme determined directly by multiple substitutions of Ile 3. Nature, 334, 406-410.

Matthews,B.W. (1987) Genetic and structural analysis of the protein stability problem. Biochemistry, 26, 6885-6888.

Millar,D.S., Wacey,A.I., Ribando,J., Melissari,E., Laursen,B., Woods,P., Kakkar,V.V. and Cooper,D.N. (1994) Three novel missense mutations in the antithrombin III (AT3) gene causing recurrent venous thrombosis. Hum. Genet., 94, 509-512.

Mitraki,A., Fane,B., Haase-Pettingell,C., Sturtevant,J. and King,J. (1991) Global suppression of protein folding defects and inclusion body formation. Science, 253, 54-58.

Mottonen,J., Strand,A., Symersky,J., Sweet,R.M., Danley,D.E., Geoghegan,K.F., Gerard,R.D. and Goldsmith,E.J. (1992) Structural basis of latency in plasminogen activator inhibitor-1. Nature, 355, 270-273.

Potempa,J., Korzus,E. and Travis,J. (1994) The serpin superfamily of proteinase inhibitors: structure, function and regulation. J. Biol. Chem., 269, 15957-15960.

Sandberg,W.S. and Terwilliger,T.C. (1993) Engineering multiple properties of a protein by combinatorial mutagenesis. Proc. Natl Acad Sci. USA, 90, 8367-8371.

Schreuder,H.A., de Boer,B., Dijkema,R., Mulders,J., Theunissen,H.J.M., Grootenhuis,P.D.J. and Hol,W.G.J. (1994) The intact and cleaved human antithrombin III complex as a model for serpin-proteinase interactions. Nature Struct. Biol., 1, 48-54.

Schulze,A.J., Huber,R., Bode,W. and Engh,R.A. (1994) Structural aspects of serpin inhibition. FEBS Lett., 344, 117-124.

Sherman,P.M., Lawrence,D.A., Yang,A.Y., Vandenberg,E.T., Paielli,D., Olson,S.T., Shore,J.D. and Ginsburg,D. (1992) Saturation mutagenesis of the plasminogen activator inhibitor-1 reactive center. J. Biol. Chem., 267, 7588-7595.

Sherman,P.M., Lawrence,D.A., Verhamme,I.M., Paielli,D., Shore,J.D. and Ginsburg,D. (1995) Identification of tPA-specific plasminogen activator inhibitor-1 mutants: Evidence that second sites of interaction contribute to target specificity J. Biol. Chem., 270, 9301-9306.

Shoichet,B.K., Baase,W.A., Kuroki,R. and Matthews,B.W. (1995) A relationship between protein stability and protein function. Proc. Natl Acad. Sci. USA, 92, 452-456.

Stein,P.E. and Carrell,R.W. (1995) What do dysfunctional serpins tell us about molecular mobility and disease? Struct. Biol., 2, 96-113.

Stein,P.E., Leslie,A.G.W., Finch,J.T., Turnell,W.G., McLaughlin,P.J. and Carrell,R.W. (1990) Crystal structure of ovalbumin as a model for the reactive centre of serpins. Nature, 347, 99-102.

Stemmer,W.P.C. (1994) Rapid evolution of a protein in vitro by DNA shuffling. Nature, 370, 389-391.

Tucker,H.M., Mottonen,J., Goldsmith,E.J. and Gerard,R.D. (1994) The structural basis of latency in PAI-1. Fibrinolysis, 8, 17 (Abstract)

Vassalli,J.-D. and Pepper,M.S. (1994) Membrane proteases in focus Nature, 370, 14-15.

Vassalli,J.-D., Sappino,A.-P. and Belin,D. (1991) The plasminogen activator/plasmin system. J. Clin. Invest., 88, 1067-1072.

Wei,A., Rubin,H., Cooperman,B.S. and Christianson,D.W. (1994) Crystal structure of an uncleaved serpin reveals the conformation of an inhibitory reactive loop. Nature Struct. Biol., 1, 251-258.

Received on March 10, 1995; revised on April 11, 1995 